\title{
Robust Satisfaction of Nonlinear Requirements in Control Problems
}

\author{
Maxime Pouilly-Cathelain ${ }^{1}$, Philippe Feyel $^{2}$, Gilles Duc ${ }^{3}$ and Guillaume Sandou ${ }^{3}$
}

\begin{abstract}
Industrial framework needs methods to take into account complex and non-differentiable requirements given by customers directly in control design for disturbed systems. The aim of this paper is to take into account any evaluable requirements in the design of control laws for systems subject to additive disturbances. To achieve this goal, Barrier Model Predictive Control and invariant set theory are used. It allows considering state and input constraints and also to improve robustness with regard to disturbances.
\end{abstract}

\section{INTRODUCTION}

In numerous control problems, considering both hard constraints and requirements on state and input is of great interest, especially in an industrial context where requirements are given by the customers. Two kinds of constraints have to be considered: those that are essential for the system to operate and those that ensure satisfactory performances, respectively named hard constraints and requirements in this paper. Moreover, the system is often subject to additive disturbances and measurements errors. Finally as the number of sensors is restricted, the whole state cannot be measured, and an observer must therefore be used.

Model Predictive Control (MPC) is generally the preferred method to take into account constraints because they are considered online, [1]. As a reminder, a sequence of future control inputs is determined at each sampling instant by online optimization over a finite horizon, while only the first one is actually applied to the system. Constraints are directly considered by the optimizer along the prediction horizon. In order to take into account disturbances, robust MPC has been proposed in [2] and [3]. Usually, robust MPC is based on tubes of robust positive invariant sets ([4], [5]) centered in the system's nominal trajectory. With this method, system's state is guaranteed to remain inside the tube, close to the nominal desired trajectory regardless of disturbances.

In this paper, it is admitted that hard constraints are satisfied thanks to a high level supervisor that generates admissible trajectories, thus only requirements will be considered in the MPC. To do so, different methods have been proposed. The first one is to formulate them as hard constraints [1], but this solution is quite restrictive as the system could operate even if requirements would temporarily be unsatisfied. A different

\footnotetext{
${ }^{1}$ Maxime Pouilly-Cathelain is with Safran Electronics \& Defense, Massy, France and L2S, CentraleSupélec, CNRS, Université Paris-Sud, Université Paris-Saclay, Gif-sur-Yvette, France maxime.pouilly-cathelain $₫$ \{centralesupelec.fr, safrangroup.com

${ }^{2}$ Philippe Feyel is with Safran Electronics \& Defense, Massy, France philippe.feyelesafrangroup.com

${ }^{3}$ Gilles Duc and Guillaume Sandou are with L2S, CentraleSupélec, CNRS, Université Paris-Sud, Université Paris-Saclay, Gif-sur-Yvette, France \{gilles.duc, guillaume.sandou\}@centralesupelec.fr
}

method based on barrier functions has been proposed in [6]: it permits to give the system the opportunity to violate requirements.

Barrier MPC stability and practical implementation have been studied in [7] and [8]. Recently, [9] proposed a barrier MPC that improves robustness with respect to unstructured model uncertainty. Nevertheless, to the best of our knowledge, there is no literature about the use of barrier function in a MPC that is robust with respect to additive disturbances.

This paper provides a method to achieve robust satisfaction of nonlinear requirements for linear systems subject to additive disturbances by using tube-based MPC with barrier functions. To do so, properties of barrier functions are presented in section II, a new formulation of the cost function is proposed in section III while section IV provides a proof of stability in the nominal case. Robust satisfaction of requirements is studied in section V. Finally, section VI gives an example where the use of an Unmanned Aerial Vehicle (UAV) for reading QR codes in a storage space with hard constraints to avoid collision and requirements to remain at the right distance of the $\mathrm{QR}$ code are considered. Conclusions and future works are given in section VII.

Notation. In the following, $\mathbb{R}$ and $\mathbb{N}$ are respectively the set of the real numbers and the set of positive integers. $\mathbb{N}^{*}=$ $\mathbb{N} \backslash\{0\}$. $\mathbb{U}$ denotes the input constraint set. If $\mathbf{A}$ is a matrix, $\rho(\mathbf{A})$ denotes its spectral radius. The norm $\|x\|_{\mathbf{Q}}^{2}=x^{\mathrm{T}} \mathbf{Q} x$ with $\mathbf{Q}$ a strictly positive definite matrix is considered. $\|x\|_{\infty}=$ $\max _{i}\left|x_{i}\right|$ denotes the infinity norm. The Minkowski sum of two sets $\mathbb{A}$ and $\mathbb{B}$ is the set $\mathbb{A} \oplus \mathbb{B}=\{a+b \mid a \in \mathbb{A}, b \in \mathbb{B}\}$. The Pontryagin difference of two sets $\mathbb{A}$ and $\mathbb{B}$ is the set $\mathbb{A} \ominus$ $\mathbb{B}=\{a \in \mathbb{A} \mid a+b \in \mathbb{A}, \forall b \in \mathbb{B}\} . d(x, \mathbb{A})=\inf \{\|x-a\|, a \in \mathbb{A}\}$ denotes the distance between vector $x$ and set $\mathbb{A}$.

\section{REQUIREMENTS CONSIDERATION}

Invariant sets are of great interest for stability of MPC. Some important definitions used in the sequel are recalled below.

Definition 1 (Admissible set, [4]): A set is an admissible set if for all elements of this set, it is possible to find an input sequence that enforces the system to reach the origin, which is the target point, without violating any hard constraint.

Definition 2 (Control Positive Invariant (CPI) set, [4]):

A set $\mathbb{S}$ is said to be a Control Positive Invariant set for the system $x_{k+1}=\mathbf{A} x_{k}+\mathbf{B} u_{k}$ and the feedback law $u_{k}=\mathbf{K} x_{k}$ if $\forall x_{k} \in \mathbb{S},(\mathbf{A}+\mathbf{B K}) x_{k} \in \mathbb{S}$.

With these two definitions, assumption 1 is done. This assumption is satisfied thanks to a high-level supervisor. As a consequence, hard constraints are not considered in this paper except for the input hard constraint $u \in \mathbb{U}$. 
Assumption 1: The state of the system always remains in an admissible set that is CPI. This set is denoted by $\mathbb{X}$.

In an ideal case, we would like to consider requirements on an infinite prediction horizon. Since it is not realistic, requirements will be considered only on predicted states and inputs, as it is usually done in MPC when constraints are introduced.

In order to prove stability, requirements have to be classified before using them as barrier functions and the set where all requirements are considered should be determined. As a first step, no disturbances are considered. They will be later introduced in section $\mathrm{V}$.

\section{A. REQUIREMENT CLASSIFICATION}

In this paper, only inequality requirements $r$ defined by (1) are considered because equality requirements can be converted into two inequalities using (2). In (1) and (2), $\mathscr{X}$ denotes a set of any predicted states and $\mathscr{U}$ a set of any elements of the input sequence.

$$
\begin{gathered}
r(\mathscr{X}, \mathscr{U}) \leqslant 0 \\
r(\mathscr{X}, \mathscr{U})=0 \rightarrow\left\{\begin{array}{l}
r(\mathscr{X}, \mathscr{U}) \leqslant 0 \\
r(\mathscr{X}, \mathscr{U}) \geqslant 0
\end{array}\right.
\end{gathered}
$$

The $N_{r}$ requirements can be classified into two categories:

1) Requirements that are applied to each predicted state independently. $N_{r}^{*}$ denotes the number of requirements that belong to this category.

2) Requirements that are applied to a set of predicted states. The number of requirements that belong to this category is $N_{r}-N_{r}^{*}$.

\section{B. DEFINITION OF THE REQUIREMENT ADMISSIBLE SET}

The maximal requirement admissible set is introduced by definition 3.

Definition 3 (Maximal Requirement Admissible set):

A set $\mathbb{M}$ is said to be Maximal Requirement Admissible (MRA) if all sets $\mathbb{A}$ where the requirements are satisfied are such that: $\mathbb{A} \subseteq \mathbb{M}$.

The construction of a MRA set $\mathbb{M}$ is now described. If for each requirement $r \in \llbracket 1 ; N_{r} \rrbracket$, the corresponding MRA set is $\mathbb{X}_{r}, \mathbb{M}$ is defined by (3). $\mathbb{X}_{r}$ can be determined exactly if the requirement is simple but, in some cases, conservative approximations have to be used.

$$
\mathbb{M}=\bigcap_{r=1}^{N_{r}} \mathbb{X}_{r}
$$

Assumption 2: All requirements are satisfied at the origin: $\forall r \in \llbracket 1 ; N_{r} \rrbracket, 0 \in \mathbb{X}_{r}$.

In order to prove stability, the MRA set $\mathbb{M}$ needs to be CPI. For this purpose, a set $\mathbb{M}_{C P I} \subseteq \mathbb{M}$ will be considered as a CPI approximation of $\mathbb{M}$. A convex CPI approximation can be defined, taking into account the constraint: $\forall k \in \mathbb{N}, u_{k} \in \mathbb{U}$, by using algorithm defined in [10].

Definition 4 is also needed for the sequel.
Definition 4 (m-backward reachable set, [11]): For all $m \in \mathbb{N}^{*}$, the $m$-backward reachable set $\operatorname{Pre}(\mathbb{S}, m)$, also named $m$-preimage set, is defined as the set from where it is possible to reach $\mathbb{S}$ in $m$ steps.

The maximal backward reachable set included in a given set can be defined using the algorithm presented in [12].

In the following, it is admitted that $\mathbb{M}_{C P I}$ is known.

\section{BARRIER FUNCTION DEFINITION}

In order to prove stability, for barrier function definition, we only consider requirements that belong to the first category presented in II-A which are requirements independently applied to each predicted state. The barrier $l_{r}$ corresponding to requirement $r$ and its properties are defined by (4). In (4), $\bar{x}_{k}$ is the predicted state for time index $k \in \llbracket 0 ; N \rrbracket$ with $N$ denoting the prediction horizon and $\mathbf{u}=\left[u_{0}, \ldots, u_{N-1}\right]$ is the input sequence determined by the MPC optimizer. Consequently, the global barrier function $l_{b}$ can be defined by (5). One can remark that properties defined for $l_{r}$ still hold for $l_{b}$.

$$
\begin{array}{r}
\forall(\mathbf{u}, k) \in \mathbb{U}^{N} \times \llbracket 0 ; N \rrbracket, \quad\left\{\begin{array}{lll}
l_{r}\left(\bar{x}_{k}, \mathbf{u}\right)=0 & \text { if } & \bar{x}_{k} \in \mathbb{X}_{r} \\
l_{r}\left(\bar{x}_{k}, \mathbf{u}\right) \geqslant 0 & \text { if } & \bar{x}_{k} \notin \mathbb{X}_{r}
\end{array}\right. \\
\forall(\mathbf{u}, k) \in \mathbb{U}^{N} \times \llbracket 0 ; N \rrbracket, \quad l_{b}\left(\bar{x}_{k}, \mathbf{u}\right)=\sum_{r=1}^{N_{r}^{*}} l_{r}\left(\bar{x}_{k}, \mathbf{u}\right)
\end{array}
$$

Since $l_{b}$ is a barrier function that may be applied to all predicted states $\overline{\mathbf{x}}=\left[\bar{x}_{0}, \ldots, \bar{x}_{N}\right]$, the notation $l_{b}(\overline{\mathbf{x}}, \mathbf{u})$ will be used in the sequel.

\section{COST FUNCTION DEFINITION}

As it is often the case in MPC, the design of the cost function has to be done in order to ensure stability, [13]. In classical MPC formulation where the cost function is used as a Lyapunov function, the cost function is needed to decrease from one sampling instant to the next one. Because this paper deals with nonlinear requirements, it cannot be ensured that the barrier function decreases in $\mathbb{X} \backslash \mathbb{M}_{C P I}$ from one sampling instant to the next one. Nevertheless, it will be shown in section IV that thanks to the CPI property of $\mathbb{M}_{C P I}$, it exists a sequence of inputs that permits, when $\bar{x}_{0} \in \operatorname{Pre}\left(\mathbb{M}_{C P I}, N\right)$, the decrease of a cost function which includes barrier functions. As a consequence, the proposed method aims to enforce the system to reach Pre $\left(\mathbb{M}_{C P I}, N\right)$ and then applies a barrier MPC. The method is thus based on two cases which correspond to the two following cost functions:

1) If the current state $x=\bar{x}_{0}$ is not in $\mathbb{M}_{C P I}$ thus the considered cost function $J_{1}$ is defined by (6). This cost function corresponds to a terminal constraint.

$$
J_{1}(\overline{\mathbf{x}}, \mathbf{u})=d\left(\bar{x}_{N}, \mathbb{M}_{C P I}\right)
$$

2) If the current state $x=\bar{x}_{0}$ is in Pre $\left(\mathbb{M}_{C P I}, N\right)$, the considered cost function $J_{2}$ is defined by (7) where $l$ denotes the MPC nominal cost: $l\left(\bar{x}_{k}, u_{k}\right)=\left\|\bar{x}_{k}\right\|_{Q}^{2}+$ $\left\|u_{k}\right\|_{R}^{2}$ and $l_{N}$ a terminal cost.

$$
J_{2}(\overline{\mathbf{x}}, \mathbf{u})=\sum_{k=0}^{N-1} l\left(\bar{x}_{k}, u_{k}\right)+l_{N}\left(\bar{x}_{N}\right)+l_{b}(\overline{\mathbf{x}}, \mathbf{u})
$$


We want to consider one single optimization problem thus a cost function $J$ that takes into account both cases is presented in $(8)$.

$$
J(\overline{\mathbf{x}}, \mathbf{u})= \begin{cases}J_{1}(\overline{\mathbf{x}}, \mathbf{u}) & \text { if } \bar{x}_{0} \notin \operatorname{Pre}\left(\mathbb{M}_{C P I}, N\right) \\ -1 / J_{2}(\overline{\mathbf{x}}, \mathbf{u}) & \text { if } \bar{x}_{0} \in \operatorname{Pre}\left(\mathbb{M}_{C P I}, N\right) \text { and } J_{2}(\overline{\mathbf{x}}, \mathbf{u}) \neq 0 \\ -\infty & \text { if } \bar{x}_{0} \in \operatorname{Pre}\left(\mathbb{M}_{C P I}, N\right) \text { and } J_{2}(\overline{\mathbf{x}}, \mathbf{u})=0\end{cases}
$$

Remark 1: From an implementation point of view, the problem is solved by using a gradient-free optimization algorithm. By using this kind of algorithm there is no need of reformulation or linearisation of requirements because non-differentiable barrier functions can be tackled and hard constraint $u \in \mathbb{U}$ is considered as the search space.

Remark 2: It is not necessary to explicitly define $\operatorname{Pre}\left(\mathbb{M}_{C P I}, N\right)$ because conditions $\bar{x}_{0} \notin \operatorname{Pre}\left(\mathbb{M}_{C P I}, N\right)$ and $\bar{x}_{0} \in \operatorname{Pre}\left(\mathbb{M}_{C P I}, N\right)$ are respectively equivalent to $\bar{x}_{N} \notin \mathbb{M}_{C P I}$ and $\bar{x}_{N} \in \mathbb{M}_{C P I}$. Moreover, $\mathbb{M}_{C P I}$ has already been defined (section II).

\section{PROOF OF STABILITY IN THE NOMINAL CASE}

In this section, a proof of stability for the proposed MPC using the cost function presented in section III is provided. To begin with, it will be shown that the system will reach Pre $\left(\mathbb{M}_{C P I}, N\right)$. Then a proof based on a Lyapunov function will be given when $\bar{x}_{0} \in \operatorname{Pre}\left(\mathbb{M}_{C P I}, N\right)$. Assumption 3 is done for clarity of the proof but in practice this assumption can be relaxed as it will be discussed in sections IV-C and V.

Assumption 3: The optimization algorithm always finds the global minimum.

\section{A. CONVERGENCE FOR $x_{k}=\bar{x}_{0} \notin \operatorname{Pre}\left(\mathbb{M}_{C P I}, N\right)$}

The cost function has been formulated in order to firstly determine a trajectory such that $d\left(\bar{x}_{N}, \mathbb{M}_{C P I}\right)=0$, but in the case where $x_{K}=\bar{x}_{0} \notin \operatorname{Pre}\left(\mathbb{M}_{C P I}, N\right)$ such a trajectory does not exist. In practice, it corresponds to a positive cost at the end of the optimization. In this case, a LQ control law is applied to the system to ensure that the system's state converges to $\operatorname{Pre}\left(\mathbb{M}_{C P I}, N\right)$.

Remark 3: The idea that consists of applying the input sequence determined by the MPC algorithm that decreases the distance from $\mathbb{M}_{C P I}$ is not sufficient to ensure convergence thus, it is not a viable solution.

\section{B. CONVERGENCE FOR $x_{k}=\bar{x}_{0} \in \operatorname{Pre}\left(\mathbb{M}_{C P I}, N\right)$}

As it is usually done in MPC, a Lyapunov function based on the cost function will be used in this section. The following function $V$ will be considered, taking into account that $\bar{x}_{0} \in \operatorname{Pre}\left(\mathbb{M}_{C P I}, N\right)$ :

$$
\forall(\overline{\mathbf{x}}, \mathbf{u}) \in\left(\operatorname{Pre}\left(\mathbb{M}_{C P I}, N\right)\right)^{N+1} \times \mathbb{U}^{N}, V(\overline{\mathbf{x}}, \mathbf{u})=\frac{-1}{J(\overline{\mathbf{x}}, \mathbf{u})}=J_{2}(\overline{\mathbf{x}}, \mathbf{u})
$$

Because $l$ and $l_{N}$ are positive functions, $V$ is a positive function. Moreover, $V(0,0)=0$.

The ${ }^{+}$notation denotes quantities at the next sampling instant, for instance the next input sequence is denoted by $\mathbf{u}^{+}$. Similarly to the warm start method presented in [14], it can be chosen: $\mathbf{u}^{+}=\left[u_{1}, \ldots, u_{N-1}, u^{+}\right]$where $u^{+} \in \mathbb{U}$ such that $\bar{x}_{N}^{+} \in \mathbb{M}_{C P I}$. The existence of such $u^{+}$is directly derived from the property of positive invariance of $\mathbb{M}_{C P I}$.

The difference $V\left(\overline{\mathbf{x}}^{+}, \mathbf{u}^{+}\right)-V(\overline{\mathbf{x}}, \mathbf{u})$ has to be evaluated.

$$
\begin{aligned}
& V\left(\overline{\mathbf{x}}^{+}, \mathbf{u}^{+}\right)-V(\overline{\mathbf{x}}, \mathbf{u}) \\
= & \sum_{k=0}^{N-1} l\left(\bar{x}_{k}^{+}, u_{k}^{+}\right)+l_{N}\left(\bar{x}_{N}^{+}\right)+l_{b}\left(\overline{\mathbf{x}}^{+}, \mathbf{u}^{+}\right) \\
& -\left[\sum_{k=0}^{N-1} l\left(\bar{x}_{k}, u_{k}\right)+l_{N}\left(\bar{x}_{N}\right)+l_{b}(\overline{\mathbf{x}}, \mathbf{u})\right] \\
= & \sum_{k=0}^{N-1}\left[l\left(\bar{x}_{k}^{+}, u_{k}^{+}\right)-l\left(\bar{x}_{k}, u_{k}\right)\right] \\
& +l_{N}\left(\bar{x}_{N}^{+}\right)-l_{N}\left(\bar{x}_{N}\right) \\
& +l_{b}\left(\overline{\mathbf{x}}^{+}, \mathbf{u}^{+}\right)-l_{b}(\overline{\mathbf{x}}, \mathbf{u})
\end{aligned}
$$

As a reminder from section II-C, only barrier functions derived from requirements applied independently to each predicted state are taken into account. Because no disturbances are considered, $\overline{\mathbf{x}}^{+}=\left[\bar{x}_{1}, \ldots, \bar{x}_{N}, \bar{x}_{N}^{+}\right]$thus $l_{b}\left(\overline{\mathbf{x}}^{+}, \mathbf{u}^{+}\right)-$ $l_{b}(\overline{\mathbf{x}}, \mathbf{u})$ can be simplified by looking only at the influence of the first component: $\bar{x}_{0}$, and the last one: $\bar{x}_{N}^{+}$. In order to make it appears explicitly, the notation $l_{b}\left(\overline{\mathbf{x}}^{+}, \mathbf{u}^{+}\right)-l_{b}(\overline{\mathbf{x}}, \mathbf{u})=$ $l_{b}\left(\bar{x}_{N}^{+}, \mathbf{u}^{+}\right)-l_{b}\left(\bar{x}_{0}, \mathbf{u}\right)$ is used in the following. Equation (10) is therefore simplified as:

$$
\begin{aligned}
V\left(\overline{\mathbf{x}}^{+}, \mathbf{u}^{+}\right)-V(\overline{\mathbf{x}}, \mathbf{u})= & l\left(\bar{x}_{N-1}^{+}, u_{N-1}^{+}\right)-l\left(\bar{x}_{0}, u_{0}\right) \\
& +l_{N}\left(\bar{x}_{N}^{+}\right)-l_{N}\left(\bar{x}_{N}\right) \\
& +l_{b}\left(\bar{x}_{N}^{+}, \mathbf{u}^{+}\right)-l_{b}\left(\bar{x}_{0}, \mathbf{u}\right)
\end{aligned}
$$

By definition of $\mathbb{M}_{C P I}, \bar{x}_{N}^{+} \in \mathbb{M}_{C P I} \Rightarrow l_{b}\left(\bar{x}_{N}^{+}, \mathbf{u}^{+}\right)=0$ thus finally:

$$
\begin{aligned}
V\left(\overline{\mathbf{x}}^{+}, \mathbf{u}^{+}\right)-V(\overline{\mathbf{x}}, \mathbf{u})= & l\left(\bar{x}_{N-1}^{+}, u_{N-1}^{+}\right)-l\left(\bar{x}_{0}, u_{0}\right) \\
& +l_{N}\left(\bar{x}_{N}^{+}\right)-l_{N}\left(\bar{x}_{N}\right) \\
& -l_{b}\left(\bar{x}_{0}, \mathbf{u}\right)
\end{aligned}
$$

$l_{b}$ is a positive function then:

$$
\begin{aligned}
& V\left(\overline{\mathbf{x}}^{+}, \mathbf{u}^{+}\right)-V(\overline{\mathbf{x}}, \mathbf{u}) \\
\leqslant & l_{N}\left(\bar{x}_{N}^{+}\right)-l_{N}\left(\bar{x}_{N}\right)+l\left(\bar{x}_{N-1}^{+}, u_{N-1}^{+}\right)-l\left(\bar{x}_{0}, u_{0}\right)
\end{aligned}
$$

The right part of inequality (13) corresponds to the classical equation that is found in the proof of stability of the original Model Predictive Control. According to [14], if we choose for instance $l_{N}\left(\bar{x}_{N}\right)=\bar{x}_{N}^{\mathrm{T}} \mathbf{P} \bar{x}_{N}$ where $\mathbf{P}$ is the positive definite solution of the Ricatti equation:

$$
\mathbf{P}=\mathbf{Q}+\mathbf{A}^{\mathrm{T}} \mathbf{P} \mathbf{A}-\mathbf{A}^{\mathrm{T}} \mathbf{P B}\left(\mathbf{B}^{\mathrm{T}} \mathbf{P B}+\mathbf{R}\right)^{-1} \mathbf{B}^{\mathrm{T}} \mathbf{P} \mathbf{A},
$$

it has been proven that:

$$
l_{N}\left(\bar{x}_{N}^{+}\right)-l_{N}\left(\bar{x}_{N}\right)+l\left(\bar{x}_{N-1}^{+}, u_{N-1}^{+}\right)-l\left(\bar{x}_{0}, u_{0}\right)<0
$$

From (13), we finally have $V\left(\overline{\mathbf{x}}^{+}, \mathbf{u}^{+}\right)-V(\overline{\mathbf{x}}, \mathbf{u})<0$. As a conclusion, $V$ is a positive decreasing function with $V(0,0)=0$ thus it is a Lyapunov function and Lyapunov stability is proven for $x_{k} \in \operatorname{Pre}\left(\mathbb{M}_{C P I}, N\right)$.

\section{STABILITY IN CASE OF SUBOPTIMAL SOLUTION}

Stabilizing conditions for MPC usually rely on assumption 3. Nevertheless, this assumption is generally impossible to satisfy in a practical use, especially in this paper where the cost function is possibly non-convex and non-differentiable 
and where a gradient-free optimization algorithm is used. Some works have been done in order to prove stability of MPC in case of suboptimal solution given by the optimizer, [15], [16]. Proposed solutions are essentially based on the warmstart method that consists of using a well-chosen initial guess obtained by the optimization result at the previous sampling instant. Cited papers propose methods to relax assumption 3 and prove stability in case of suboptimal solution. The goal of this paper is not to demonstrate the practical use of the method but the ability of the method to robustly satisfy requirements thus this section only gives an overview of how the suboptimal method could be implemented in practice, but does not provide all details.

For the first case: $x_{k}=\bar{x}_{0} \notin \operatorname{Pre}\left(\mathbb{M}_{C P I}, N\right)$ (section IV-A), it is still possible to apply a linear feedback law. This law ensures that the state converges to Pre $\left(\mathbb{M}_{C P I}, N\right)$.

For the second case: $x_{k}=\bar{x}_{0} \in \operatorname{Pre}\left(\mathbb{M}_{C P I}, N\right)$ (section IVB), method proposed by [15] can be extended to the barrier function proposed in this paper to relax assumption 3 . The cost function $J_{2}$ is modified as: $J_{2}(\overline{\mathbf{x}}, \mathbf{u})=\sum_{k=0}^{N-1} l\left(\bar{x}_{k}, u_{k}\right)+l_{b}(\overline{\mathbf{x}}, \mathbf{u})$ and the stability is guaranteed using the terminal constraint: $\bar{x}_{N}=0,[1]$. Because $\{0\} \subseteq \mathbb{M}$ implies that the $N$-backward reachable set of $\{0\}$ is included or equal to Pre $\left(\mathbb{M}_{C P I}, N\right)$, then we have to consider prediction horizon of length $M>N$ in order to be able to find a solution such that: $\bar{x}_{M}=0$. Following algorithm of [15], this recursive method is used:

- Choose $\mu \in(0,1]$

- At time $k=0$, find a control sequence $\mathbf{u}=\left[u_{0}, \ldots, u_{M-1}\right]$ such that $\bar{x}_{M}=0$.

- For time $k>0$, find a control sequence $\mathbf{u}^{+}$ such that $\bar{x}_{M}=0$ and $J\left(\overline{\mathbf{x}}^{+}, \mathbf{u}^{+}\right) \leqslant J(\overline{\mathbf{x}}, \mathbf{u})-$ $\mu\left[l\left(\bar{x}_{k-1}, u_{k-1}\right)+l_{b}\left(\bar{x}_{k-1}, u_{k-1}\right)\right]$. The optimization algorithm should be initialized with $\mathbf{u}^{+}=\left[u_{1}, \ldots, u_{M-1}, 0\right]$.

The optimization problem becomes easier by choosing a value of $\mu$ close to 0 . As the author of [15] says, in some cases this algorithm may fail to find a solution that respects $J\left(\overline{\mathbf{x}}^{+}, \mathbf{u}^{+}\right) \leqslant J(\overline{\mathbf{x}}, \mathbf{u})-\mu\left[l\left(\bar{x}_{k-1}, u_{k-1}\right)+l_{b}\left(\bar{x}_{k-1}, u_{k-1}\right)\right]$. In this case there is no guarantee of stability.

\section{ROBUST SATISFACTION OF REQUIREMENTS USING TUBE-BASED MPC}

In this section, the MPC with the cost function proposed above is extended to output tube-based MPC. The system considered is subject to additive disturbances as shown in (16).

$$
\left\{\begin{aligned}
x_{k+1} & =\mathbf{A} x_{k}+\mathbf{B} u_{k}+w_{k} \\
y_{k} & =\mathbf{C} x_{k}+v_{k}
\end{aligned}\right.
$$

where, for all $k \in \mathbb{N}, x_{k} \in \mathbb{X} \subseteq \mathbb{R}^{n}, u_{k} \in \mathbb{U} \subseteq \mathbb{R}^{p}, y_{k} \in \mathbb{R}^{m}$, $w_{k} \in \mathbb{W}$ and $v_{k} \in \mathbb{V} . \mathbb{W}$ and $\mathbb{V}$ are two compact sets with 0 in their interior. Assumption 4 is done. As said before, the whole state cannot be measured thus an observer has to be designed in order to initialize the prediction. This observer is a discrete-time Luenberger observer defined by (17) where $\mathbf{L} \in \mathbb{R}^{n \times m}$.

$$
\left\{\begin{aligned}
\hat{x}_{k+1} & =\mathbf{A} \hat{x}_{k}+\mathbf{B} u_{k}+\mathbf{L}\left(y_{k}-\hat{y}_{k}\right) \\
\hat{y}_{k} & =\mathbf{C} \hat{x}_{k}
\end{aligned}\right.
$$

Assumption 4: A is non-singular, $(\mathbf{A}, \mathbf{B})$ is controllable and $(\mathbf{A}, \mathbf{C})$ is observable.

The tube-based MPC strategy presented in [2] consists of applying the MPC method on a nominal predictor defined by (18) that gives the nominal input sequence $\overline{\mathbf{u}}$. The nominal input $\overline{u_{k}}$ is summed with a feedback law according to (19) where $\mathbf{K} \in \mathbb{R}^{p \times n}$.

$$
\begin{gathered}
\forall k \in \llbracket 0 ; N-1 \rrbracket,\left\{\begin{array}{r}
\bar{x}_{k+1}=\mathbf{A} \bar{x}_{k}+\mathbf{B} \bar{u}_{k} \\
\bar{y}_{k}=\mathbf{C} \bar{x}_{k}
\end{array}\right. \\
u_{k}=\bar{u}_{k}+\mathbf{K} e_{k} \quad \text { where } e_{k}=\hat{x}_{k}-\bar{x}_{k}
\end{gathered}
$$

(19) guarantees that the system's state remains in a tube centered on the nominal trajectory. In order to define this tube we introduce the estimation error: $\tilde{x}_{k}=x_{k}-\hat{x}_{k}$ and define Robust Positive Invariant set (definition 5).

Definition 5 (Robustly Positive Invariant (RPI) set, [11]): For the autonomous discrete time system $x_{k+1}=\mathbf{A} x_{k}+w_{k}$, a set $\mathbb{S}$ is said to be Robustly Positive Invariant if for all $x_{k} \in \mathbb{S}$ and $w_{k} \in \mathbb{W}, x_{k+1} \in \mathbb{S}$. This condition is equivalent to $\mathbf{A} \mathbb{S} \oplus \mathbb{W} \subseteq \mathbb{S}$.

$e_{k}$ and $\tilde{x}_{k}$ respectively follow the difference equations (20) and (21).

$$
\begin{gathered}
e_{k+1}=(\mathbf{A}+\mathbf{B K}) e_{k}+\overline{\boldsymbol{\delta}}_{k} \quad \text { where } \quad \overline{\boldsymbol{\delta}}_{k}=\mathbf{L} \mathbf{C} \tilde{x}_{k}+\mathbf{L} v_{k} \\
\tilde{x}_{k+1}=(\mathbf{A}-\mathbf{L C}) \tilde{x}_{k}+\tilde{\delta}_{k} \quad \text { where } \quad \tilde{\boldsymbol{\delta}}_{k}=w_{k}-\mathbf{L} v_{k}
\end{gathered}
$$

According to [5], if $\rho(\mathbf{A}+\mathbf{B K})<1$ and $\rho(\mathbf{A}-\mathbf{L C})<1$ then RPI sets $\overline{\mathbb{S}}$ and $\widetilde{\mathbb{S}}$ exist and are finite time computable respectively for (20) and (21). Thanks to assumption 4, it is possible to find $\mathbf{K}$ and $\mathbf{L}$ such that $\rho(\mathbf{A}+\mathbf{B K})<1$ and $\rho(\mathbf{A}-\mathbf{L} \mathbf{C})<1$. For instance, $\mathbf{K}$ and $\mathbf{L}$ can be determined by using LQG design or pole placement method.

It has been proven in [2], that if $\tilde{x}_{k}=x_{k}-\hat{x}_{k} \in \tilde{\mathbb{S}}$, $e_{k}=\hat{x}_{k}-\bar{x}_{k} \in \overline{\mathbb{S}}$ and the control law is given by (19) then, using the fact that the system's state follows $x=\bar{x}+\tilde{x}+e$, for all $i \in \mathbb{N}$ and all admissible disturbance $w_{k+i} \in \mathbb{W}$ and $v_{k+i} \in \mathbb{V}, x_{k+i} \in\left\{\hat{x}_{k+i}\right\} \oplus \tilde{\mathbb{S}} \subseteq\left\{\bar{x}_{k+i}\right\} \oplus \tilde{\mathbb{S}} \oplus \overline{\mathbb{S}}$. Finally, the state $x_{k}$ follows:

$$
x_{k} \in\left\{\hat{x}_{k}\right\} \oplus \tilde{\mathbb{S}} \subseteq\left\{\bar{x}_{k}\right\} \oplus \mathbb{S} \quad \text { where } \quad \mathbb{S}=\tilde{\mathbb{S}} \oplus \overline{\mathbb{S}}
$$

Because the input follows (19), a tighter constraint has to be applied to the nominal input $\bar{u}$ to ensure that: $u \in \mathbb{U}$. This tighter constraint is defined by (23). In order to ensure that requirements are also robustly satisfied, the set $\mathbb{M}$ defined by (3) has to be restricted to $\mathbb{M}^{-}=\mathbb{M} \ominus \mathbb{S}$. $\mathbb{M}_{C P I}^{-} \subseteq \mathbb{M}_{C P I}$ denotes the CPI set included in $\mathbb{M}^{-}$by considering $\overline{\mathbb{U}}$ rather than $\mathbb{U}$.

$$
\bar{u} \in \overline{\mathbb{U}} \quad \text { where } \quad \overline{\mathbb{U}}=\mathbb{U} \ominus \mathbf{K} \overline{\mathbb{S}}
$$

Tighter requirements also have to be considered for barrier function definition. For each requirement $r$, the set $\mathbb{X}_{r}$ considered in (4) must be replaced by $\mathbb{X}_{r} \ominus \mathbb{S}$.

Remark 4: As a reminder from [2], robust control by using tube-based MPC can only be achieved if $\mathbb{W}$ and $\mathbb{V}$ are sufficiently small because the set $\mathbb{S}$ becomes larger when $\mathbb{W}$ or $\mathbb{V}$ grow. 
Remark 5: The suboptimal MPC method briefly introduced in section IV-C is not necessary when tube-based MPC is used because small optimization error could be seen as disturbances. For instance if the optimal input is $u_{k}^{*}$ but the algorithm finds $u_{k}^{*}+u_{k}^{e}$, then $x_{k+1}=\mathbf{A} x_{k}+\mathbf{B}\left(u_{k}^{*}+u_{k}^{e}\right)$ and by choosing $w_{k}=\mathbf{B} u_{k}^{e}$, the equation corresponds to the one used in tube-based MPC framework (16). The only assumption is that $u^{e}$ is bounded. Thanks to the warm start method, this is in practice often true.

\section{APPLICATION}

As said in the introduction, this methodology could be applied to UAVs used to read QR codes in a storage space. Because only altitude control is considered in this application, the system is simply modelled by (24) where all values have been normalized. Position and velocity are respectively denoted by $z$ and $v^{z}$ thus in (24), $x_{k}=\left[z_{k}, v_{k}^{z}\right]^{\mathrm{T}}$. The system is subject to uniform additive disturbances $w_{k} \in \mathbb{W}$ and $v_{k} \in$ $\mathbb{V}$ where $\mathbb{W}=\left\{w \in \mathbb{R}^{2},|| w \|_{\infty} \leqslant 0.1\right\}$ and $\mathbb{V}=\{v \in \mathbb{R},|v| \leqslant$ $0.05\} . w$ and $v$ model external disturbances such as airstreams, measurement errors and suboptimality of the optimizer (see remark 5).

$$
\left\{\begin{aligned}
x_{k+1} & =\left[\begin{array}{ll}
1 & 1 \\
0 & 1
\end{array}\right] x_{k}+\left[\begin{array}{l}
1 \\
1
\end{array}\right] u_{k}+w_{k} \\
y_{k} & =\left[\begin{array}{ll}
1 & 0
\end{array}\right] x_{k}+v_{k}
\end{aligned}\right.
$$

Hard constraints such as collision avoidance are considered to be satisfied thanks to a high-level supervisor. The only exception is the input constraint: $u \in \mathbb{U}=\{u \in \mathbb{R},|u| \leqslant 3\}$ that is used as the search space of the optimization algorithm that will be differential evolution, [17]. Nevertheless, in order to be able to read the QR code, the following requirements have to be considered regardless of the disturbances:

- The UAV must stay with an absolute position less than 6 (normalized unit). The target point corresponds to $z=0$.

- The UAV speed must have a variance less than 12 (normalized unit) in order to avoid blurred picture.

The first requirement leads exactly to the set $\mathbb{X}_{z}=\left\{\left[z, v^{z}\right]^{\mathrm{T}} \in\right.$ $\left.\mathbb{R}^{2},|z| \leqslant 6\right\}$ and for the second requirement the conservative set $\mathbb{X}_{v^{z}}=\left\{\left[z, v^{z}\right]^{\mathrm{T}} \in \mathbb{R}^{2},\left|v^{z}\right| \leqslant 6\right\}$ could be considered. Remind that only the first requirement acts for the definition of a barrier function whereas both requirements are used to define the requirement admissible set $\mathbb{M}=\mathbb{X}_{z} \cap \mathbb{X}_{y^{z}}=\{x \in$ $\left.\mathbb{R}^{2},\|x\|_{\infty} \leqslant 6\right\}$ which is not maximal requirement admissible.

Only the position can be measured thus a Luenberger observer (17) tuned by pole placement is used with the gain $\mathbf{L}=\left[\begin{array}{ll}1.3 & 0.42\end{array}\right]^{\mathrm{T}}$. The gain $\mathbf{K}$, of the feedback law defined in (19), is tuned using linear quadratic method, the solution is $\mathbf{K}=\left[\begin{array}{ll}-0.61 & -0.99\end{array}\right]$. One can check that $\rho(\mathbf{A}+\mathbf{B K})<1$ and $\rho(\mathbf{A}-\mathbf{L C})<1$.

The method presented above is now applied to the system. First of all, after defining $\tilde{\mathbb{S}}, \overline{\mathbb{S}}$ and $\mathbb{S}$ using the method presented in [5], the set $\mathbb{M}^{-}$is defined. The maximal positive invariant set $\mathbb{M}_{C P I}^{-} \subseteq \mathbb{M}^{-}$is computed using algorithm presented in [10]. Figures 1 and 2 represent all these sets. Figures 1 also presents Pre $\left(\mathbb{M}_{C P I}^{-}, N\right)$, the $N$-backward reachable set included in $\mathbb{M}^{-}$. By using (23), the tighter input constrained set defined for the MPC applied to the nominal system is $\bar{U}=[-1.85 ; 1.85]$. The barrier function corresponding to the absolute position error is tightened by using $\mathbb{S}$ projections onto the $z$ direction, denoted by $p(\mathbb{S}, z)$, in order to use tighter requirement (see Fig. 2) on the nominal system. The barrier function $l_{b}$ is defined by (25) where $\lim _{z}=6-\max (p(\mathbb{S}, z))$ and $\alpha=10^{5}$ is a tuning parameter.

$$
l_{b}(\overline{\mathbf{x}})=\sum_{k=1}^{N}\left\{\begin{array}{lll}
0 & \text { if } & \left|\bar{z}_{k}\right| \leqslant \lim _{z} \\
\alpha|| \bar{z}_{k}\left|-\lim _{z}\right| & \text { if } & \left|\bar{z}_{k}\right|>\lim _{z}
\end{array}\right.
$$

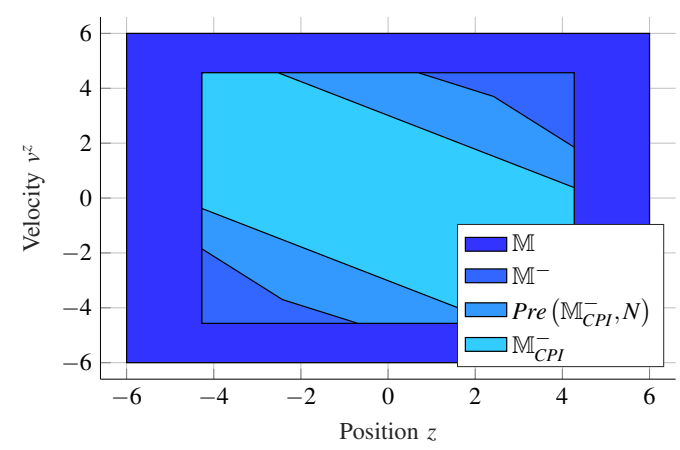

Fig. 1. Sets where all requirements are satisfied

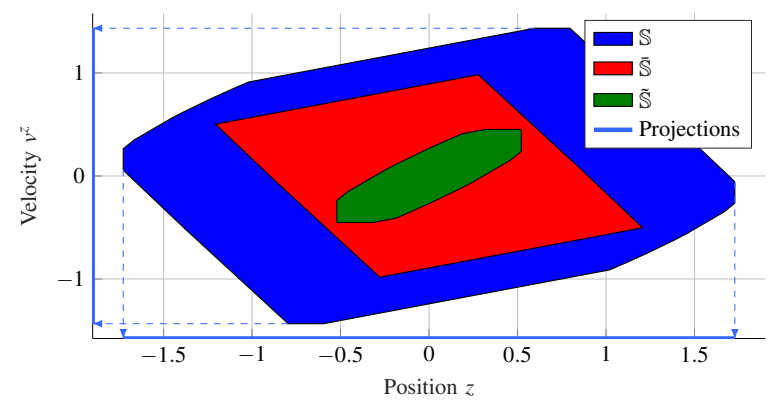

Fig. 2. Sets $\mathbb{S}, \overline{\mathbb{S}}$ and $\widetilde{\mathbb{S}}$ and $\mathbb{S}$ projections onto different directions

Figure 3 presents a trajectory of the system (24) using a tube-based MPC without requirements. In comparison, Fig. 4 presents a trajectory using the tube-based MPC proposed in this paper that takes into account requirements as barrier functions. The initial point has been chosen in Pre $\left(\mathbb{M}_{C P I}^{-}, N\right) \backslash \mathbb{M}_{C P I}^{-}$. It can be seen that the use of barrier functions permits to find a trajectory that respects requirements, which is not the case without barrier functions. In order to be fairly compared, both simulations have been done with the exact same sequence of disturbances. In order to evaluate the influence of the disturbances, Fig. 5 shows results of 75 runs from the same initial point. On the left part, results are given using a tube-based approach without requirements while the right part corresponds to the proposed method. We can see that in every runs, the proposed barrier tube-based MPC permits to respect requirements while in some cases requirements are not satisfied without using barrier functions. Of course, requirements are generally satisfied at the expense of the input. 


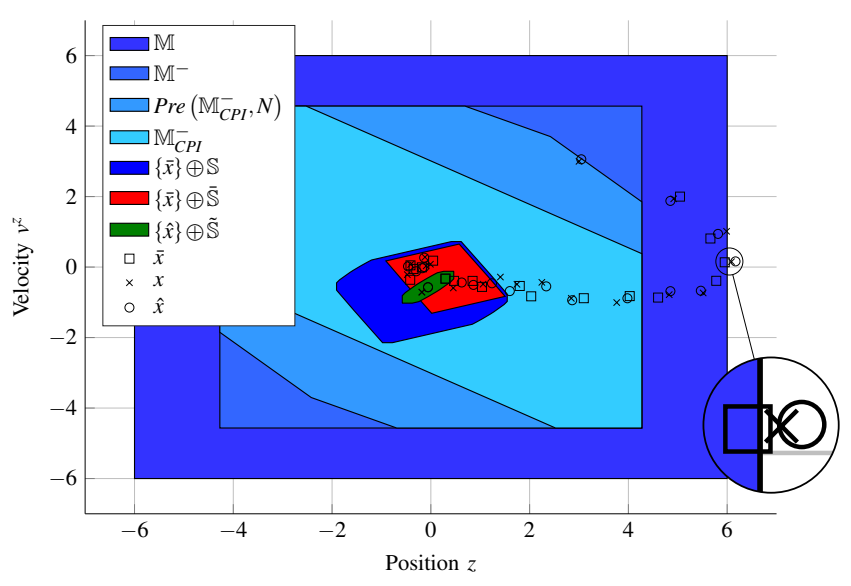

Fig. 3. Example of trajectory with tube-based MPC not using barrier functions

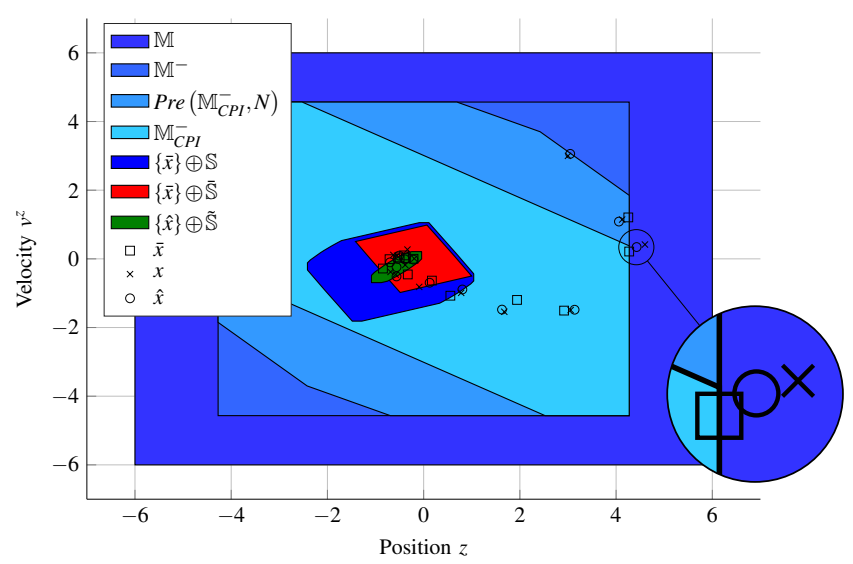

Fig. 4. Example of trajectory with tube-based MPC using barrier functions

\section{CONCLUSIONS AND FUTURE WORKS}

A solution to robustly satisfy nonlinear requirements with regard to additive disturbances and the corresponding proof of stability have been proposed. This method is based on the use of a particular cost function and a positive invariant set where requirements are satisfied. Moreover this approach has been successfully applied to an industrial context.

In future works, extension of this paper to nonlinear systems will be studied. Another possibility could be the use of barrier functions in a stochastic MPC that is generally less conservative than the tube-based approach.

\section{REFERENCES}

[1] D. Q. Mayne, J. B. Rawlings, C. V. Rao, and P. O. Scokaert, "Constrained model predictive control: Stability and optimality." Automatica, vol. 36, no. 6, pp. 789-814, 2000.

[2] D. Q. Mayne, S. Raković, R. Findeisen, and F. Allgöwer, "Robust output feedback model predictive control of constrained linear systems." Automatica, vol. 42, no. 7, pp. 1217-1222, 2006.

[3] P. O. Scokaert and D. Q. Mayne, "Min-max feedback model predictive control for constrained linear systems." IEEE Transactions on Automatic Control, vol. 43, no. 8, pp. 1136-1142, 1998.

[4] F. Blanchini, "Set invariance in control." Automatica, vol. 35, no. 11, pp. 1747-1767, 1999.

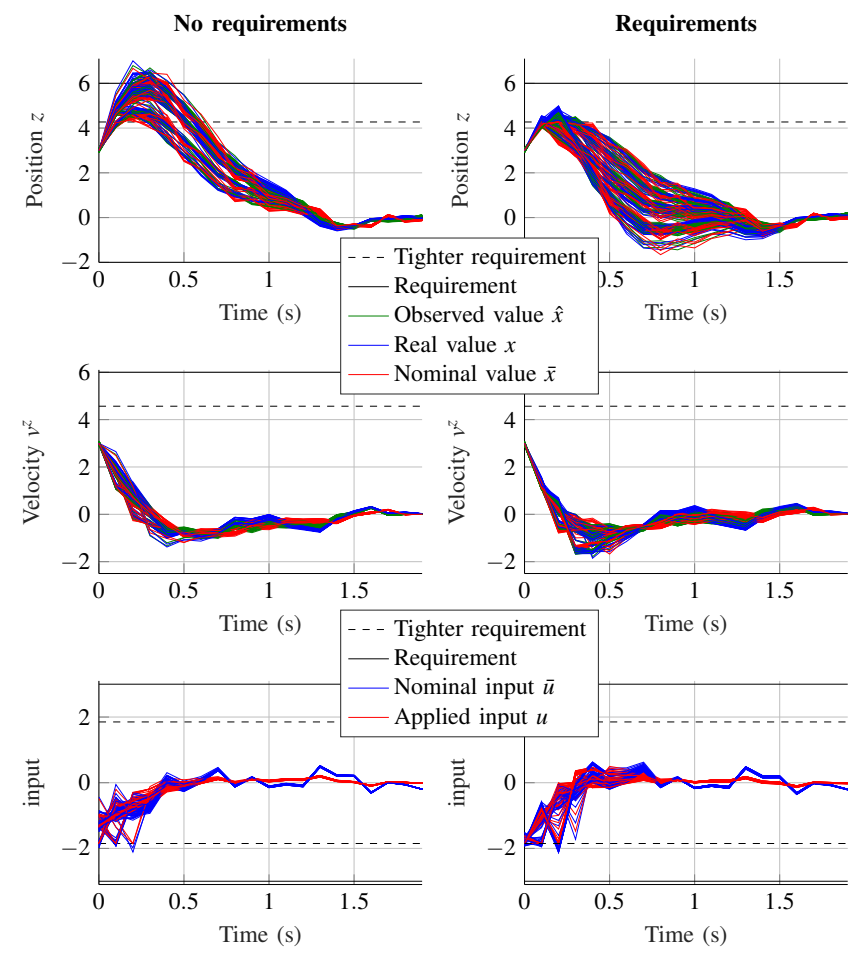

Fig. 5. Comparison of 75 runs without requirements (left) and with requirements (right)

[5] S. V. Rakovic, E. C. Kerrigan, K. I. Kouramas, and D. Q. Mayne, "Invariant approximations of the minimal robust positively invariant set." IEEE Transactions on Automatic Control, vol. 50, no. 3, pp. 406-410, 2005.

[6] A. G. Wills and W. P. Heath, "Barrier function based model predictive control." Automatica, vol. 40, no. 8, pp. 1415-1422, 2004.

[7] C. Feller and C. Ebenbauer, "Weight recentered barrier functions and smooth polytopic terminal set formulations for linear model predictive control," in 2015 American Control Conference (ACC). IEEE, 2015, pp. $1647-1652$.

[8] - "A stabilizing iteration scheme for model predictive control based on relaxed barrier functions," Automatica, vol. 80, pp. 328-339, 2017.

[9] P. Petsagkourakis, W. P. Heath, J. Carrasco, and C. Theodoropoulos, "Input-output stability of barrier-based model predictive control," 2019.

[10] E. G. Gilbert and K. T. Tan, "Linear systems with state and control constraints: The theory and application of maximal output admissible sets." IEEE Transactions on Automatic Control, vol. 36, no. 9, pp. 1008-1020, 1991.

[11] F. Blanchini and S. Miani, Set-theoretic methods in control. Springer, 2008.

[12] C. E. T. Dorea and J. Hennet, "(A,B)-invariant polyhedral sets of linear discrete-time systems," Journal of Optimization Theory and Applications, vol. 103, no. 3, pp. 521-542, 1999.

[13] J. B. Rawlings and K. R. Muske, "The stability of constrained receding horizon control," IEEE Transactions on Automatic Control, vol. 38, no. 10, pp. 1512-1516, 1993.

[14] J. B. Rawlings, D. Q. Mayne, and M. Diehl, Model Predictive Control: Theory, Computation, and Design. Nob Hill Publishing, 2017.

[15] P. O. Scokaert, D. Q. Mayne, and J. B. Rawlings, "Suboptimal model predictive control (feasibility implies stability)," IEEE Transactions on Automatic Control, vol. 44, no. 3, pp. 648-654, 1999.

[16] D. A. Allan, C. N. Bates, M. J. Risbeck, and J. B. Rawlings, "On the inherent robustness of optimal and suboptimal nonlinear MPC," Systems \& Control Letters, vol. 106, pp. 68 - 78, 2017.

[17] K. Price, R. M. Storn, and J. A. Lampinen, Differential evolution: a practical approach to global optimization. Springer Science \& Business Media, 2006. 